\title{
Flare-generated shock evolution and geomagnetic storms during the "Halloween 2003 epoch": 29 October to 2 November
}

\author{
Chin-Chun Wu, ${ }^{1}$ S. T. Wu, ${ }^{1}$ M. Dryer, ${ }^{2,3}$ C. D. Fry, ${ }^{3}$ D. Berdichevsky, ${ }^{4,5}$ Z. Smith, ${ }^{2}$ \\ T. Detman, ${ }^{2}$ N. Gopalswamy, ${ }^{5}$ R. Skoug, ${ }^{6}$ T. Zurbuchen, ${ }^{7}$ and C. Smith $^{8}$
}

Received 7 January 2005; revised 3 May 2005; accepted 19 May 2005; published 17 September 2005.

[1] The October/November 2003 ("Halloween 2003") epoch of intense solar flares provided an opportunity to test the results of earlier parametric 1.5 MHD studies of interacting interplanetary shock waves. These preliminary studies used an adaptive numerical grid that made it possible to identify products of these interactions. During 28 October to 2 November 2003, three shocks generated by four solar flares were observed at the L1 libration point by ACE/SWEPAM/SWICS/MAG. Two very distinct geomagnetic storms, associated with two of these flares (X17/4B and X10/2B), rank as two of the largest storms of solar cycle 23. The purpose of this paper is to present the use of an adaptive grid 1.5-dimensional MHD model that is initiated at the solar surface to study in detail the three shocks observed at L1 that were generated by the four solar flares. Accordingly, four separate pressure pulses, at the appropriate times and with different strengths and duration, determined via a trial and error procedure, are introduced on the Sun to mimic the four flares. The results show that the simulated solar wind velocity temporal profiles successfully matched the observations at L1. The major objective, to demonstrate the detailed nature of interacting shocks and some of their products after origination from closely spaced solar events, is achieved. In addition, the MHD model is able to suggest the solar sources that are associated with specific geomagnetic storms at Earth.

Citation: Wu, C.-C., et al. (2005), Flare-generated shock evolution and geomagnetic storms during the "Halloween 2003 epoch": 29 October to 2 November, J. Geophys. Res., 110, A09S17, doi:10.1029/2005JA011011.

\section{Introduction}

[2] Interactions of interplanetary shock waves and identification of their subsequent products are fundamentally important scientific and space weather topics. We would like to investigate the products of these interactions (whether they include slow forward and/or reverse shocks, contact discontinuities, etc.) and their survival history [Whang and Burlaga, 1986; Smith et al., 1986; C.-C. Wu et al., 1996a, 1996b, 2004, 2005a; Smith et al., 2002]. From a space weather perspective, we would like to track a specific solar event's plasma and magnetic field output as it propagates into interplanetary space with a possible geo-

\footnotetext{
${ }^{1}$ Center for Space Plasma and Aeronomic Research, University of Alabama, Huntsville, Alabama, USA.

${ }^{2}$ Space Environment Center, NOAA, Boulder, Colorado, USA.

${ }^{3}$ Exploration Physics International, Inc., Huntsville, Alabama, USA.

${ }^{4}$ L-3 Communications EER Systems, Inc., Largo, Maryland, USA.

${ }^{5}$ NASA Goddard Space Flight Center, Greenbelt, Maryland, USA.

${ }^{6}$ Los Alamos National Laboratory, Los Alamos, New Mexico, USA.

${ }^{7}$ Department of Atmospheric, Oceanic, and Space Sciences, University of Michigan, Ann Arbor, Michigan, USA.

${ }^{8}$ Institute for Earth, Oceans, and Space, University of New Hampshire, Durham, New Hampshire, USA.
}

Copyright 2005 by the American Geophysical Union. 0148-0227/05/2005JA011011 effective consequence. This latter objective is especially important for forecasting purposes when staccato solar events take place. The October/November ("Halloween 2003") epoch provided an exceptional case in point. The near real time effort [Dryer et al., 2004] to predict the shock times of arrival at L1 presents an example of this operationally imperative objective. An extensive overview of the Halloween solar-heliospheric events is provided by Veselovsky et al. [2004].

[3] Briefly, many flares and coronal mass ejections (CMEs), both halo and partial halo, were observed during late October and early November 2003. We will be concerned with only four of them. In particular, two of these on 28 and 29 October (classified in soft X rays and optical $H \alpha$ as $\mathrm{X} 17 / 4 \mathrm{~B}$ and $\mathrm{X} 10 / 2 \mathrm{~B}$, respectively) were accompanied by very high-speed halo CMEs. A major geomagnetic storm (Dst $=-363 \mathrm{nT} ; \mathrm{Ap}=252$, with a $\mathrm{Bz}=-33 \mathrm{nT}$ for a prolonged period) was "caused" by the first event. The second solar event, "aided and abetted" by this storm, induced another major storm $(D s t=-401 \mathrm{nT} ; \mathrm{Ap}=220$, with a similar Bz polarity for a slightly shorter period). These storms are the second and fourth largest of solar cycle 23. The index, Dst, of geomagnetic activity for this period is shown in Figure 1. For comparison, the largest storm in this cycle took place on 20 November 2003, with $D s t=-472 \mathrm{nT}$, following an M3.2/2N flare at N00E18 on 18 November 


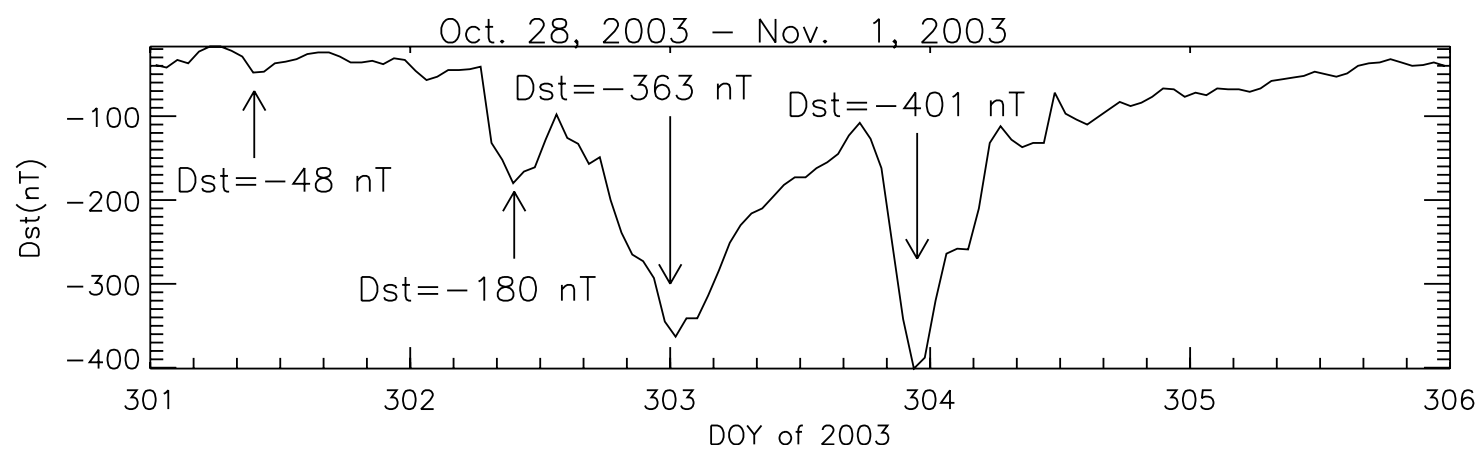

Figure 1. Geomagnetic activity Dst index during 28 October to 1 November 2003 (Kyoto University).

2003. Also, for a more recent comparison, an M9.3/2N flare at N09E05 on 6 November 2004, with a full halo CME, "caused" a geomagnetic storm on 7 November 2004, with Dst $=-383 \mathrm{nT} ; \mathrm{Ap}=220$, with a Bz varying between $-35 \mathrm{nT}$ to $-50 \mathrm{nT}$ for about 6 hours. Two days later, 910 November 2004, a similar solar flare and geomagnetic storm took place with similar Dst, $A p$, and $\mathrm{Bz}$ behaviors.

[4] Our point in mentioning these other incidents is to emphasize the staccato nature of major solar-terrestrial events and the need to study the complex nature of the propagating shocks and the products that they might spawn via interactions. In the case of Halloween 2003, there were many other flares that were observed before and after the two extreme cases mentioned at the start of the paragraph above. We will consider these two cases together with two earlier flares as backdrops for our shock interaction study.

[5] Fast forward shocks have been the most frequently observed type of shock since Gold [1955] suggested their existence in the solar wind. When a forward and a reverse fast shock propagate toward each other, a collision of the two shocks takes place in the heliosphere. Generally, the strength of both shocks is, individually, weakened [Whang and Burlaga, 1986]. An example is the case when a fast forward shock overtakes and interacts with a fast reverse shock from a preceding event [Smith et al., 1986]. The development of a time-dependent model of heliospheric interplanetary magnetic and solar wind flows [e.g., Panitchob, 1987; Panitchob et al., 1987] has made it possible to investigate a number of scientific questions concerning the evolution of solargenerated disturbances and their heliospheric consequences. Thus using this 1.5 MHD simulation model, $W u$ et al. [1996b] showed that a positive, square-wave perturbation will normally generate a pair (forward and reverse) of fast mode shocks. C.-C. Wu et al. [2004, 2005a] also demonstrated the development of slow forward and reverse shocks within the spatially larger fast forward/reverse shock pair. These studies also showed how any of these shocks may be eliminated during the complex interaction process. The results of Wu et al. [2005b] also demonstrated that the shock arrival time at Earth depends on the background solar wind speeds, the initial speeds of solar disturbances, their size, and their source location on the Sun relative to Earth's central meridian.

[6] There were at least 19 flares and CMEs observed during late October and early November 2003 [e.g., Dryer et al., 2004]. We note that not all of these events were earthward directed and thus not all would have been expected to produce effects at the Earth. For example, only eight clear fast-mode forward shocks were observed by ACE at the L1 libration point [Skoug et al., 2004]. Generally, a solar disturbance might produce a shock wave at Earth a few days later. In the present context, Dryer et al. [2004] and Smith et al. [2002, 2004] showed why some of these original solar-generated shocks decayed to fast-mode MHD waves on the portions directed toward Earth. In this study, we will use the Panitchob [1987] and the Panitchob et al. [1987] code to study postshock evolution. Shock interactions within $1 \mathrm{AU}$ following four flares during the Halloween 2003 epoch will be the focus of this study. This simulation will include a representative background solar wind with a classic Parker profile of velocity, etc. The timedependent evolution of the disturbances will extend from the Sun $\left(1 R_{\odot}\right.$, where $R_{\odot}=$ solar radius, $\left.6.95 \times 10^{5} \mathrm{~km}\right)$ to $400 R_{\odot}$ in order to allow the fourth disturbance to pass the Earth at $1 \mathrm{AU}\left(215 R_{\odot}\right)$.

[7] Section 2 outlines the mathematical model; the observations at L1 are briefly described in section 3 . The 1.5-dimensional (1.5-D) MHD model's simulation results are described in section 4 . A discussion and conclusions are presented, respectively, in sections 5 and 6.

\section{Magnetohydrodynamics Equations}

[8] The large-scale dynamics in the interplanetary solar wind can be theoretically modeled by the following MHD equations [Priest, 1982; Hughes and Brighton, 1967] in SI units:

$$
\begin{gathered}
\frac{D \rho}{D t}+\rho \nabla \cdot \mathbf{V}=0 \\
\rho \frac{D \mathbf{V}}{D t}=-\nabla p+\frac{1}{\mu_{\circ}}(\nabla \times \mathbf{B}) \times \mathbf{B}-\rho \frac{G M(r)}{r^{2}} \hat{\mathbf{r}} \\
\frac{\partial}{\partial t}\left[\rho e+\frac{1}{2} \rho|\mathbf{V}|^{2}+\frac{|\mathbf{B}|^{2}}{2 \mu_{\circ}}\right]+\nabla \cdot\left[\mathbf{V}\left\{\rho e+\frac{1}{2} \rho|\mathbf{V}|^{2}+p\right\}\right. \\
\left.+\frac{\mathbf{B} \times(\mathbf{V} \times \mathbf{B})}{\mu_{\circ}}\right]=-\mathbf{V} \cdot \rho \frac{G M(r)}{r^{2}} \hat{\mathbf{r}} \\
\frac{\partial \mathbf{B}}{\partial t}=\nabla \times(\mathbf{V} \times \mathbf{B}),
\end{gathered}
$$


Table 1. Characteristics of the Four Flares

\begin{tabular}{lccc}
\hline Occurrence of Flare $^{\mathrm{a}}$ & Class & Location & $\Delta t$, hours \\
\hline 0552 UT, 2003-10-25 & LDE M1.7/SF & N00W15 & 0 \\
0617 UT, 2003-10-26 & LDE X1.2/3N & S18E33 & 24.42 \\
1102 UT, 2003-10-28 & X17/4B & S15E08 & 77.0 \\
2041 UT, 2003-10-29 & X10/2B & S15W02 & 110.67 \\
\hline
\end{tabular}

${ }^{\text {a }}$ The flare time is listed as the radio metric Type II start time that is close to the maximum of the soft X-ray emission in 1-8A.

where $\frac{D}{D t}$ denotes the total derivative, $\rho$ is the mass density, $\mathbf{V}$ is the velocity of the flow, $p$ is the isotropic gas pressure, B is the magnetic field, $G M(r)$ is the gravitation force of Sun, $e$ is the internal energy per unit mass $(e=p /(\gamma-1) \rho)$, and $\gamma$ is the specific heat ratio. Equations (1), (2), and (3) express the conservation of mass, momentum, and energy, respectively. In Equation (2), we have ignored the viscous, heat conduction, and wave damping terms. Equation (4) is the induction equation for a perfectly conducting medium. The formulation is then closed by the ideal gas law $p=$ $2 \rho R T$ and the divergence free magnetic field, $\nabla \cdot \mathbf{B}=0$. Here, $R$ is the gas constant, $T$ is the isotropic plasma temperature, and a factor of 2 is due to the fully ionized nature of the hydrogen solar wind plasma. The detailed description of the model is given by Panitchob [1987] and Panitchob et al. [1987] and is briefly summarized in Appendix A.

\section{Observations}

\subsection{Solar Flares}

[9] A summary of the four chosen flares during the period from 25 October to 29 October 2003 is given in Table 1 . The first one took place at 0552 UT, 25 October in AR0484 at N00W15 as a long duration event (LDE) M1.7/SF flare. The second one occurred at 0617 UT, 26 October in AR0486 at S18E33 as another LDE X1/3N flare. The third one was observed at 1102 UT, 28 October, also in AR0486 at S15E08, as the X17/4B flare, followed by a halo CME as mentioned earlier. Finally, the fourth one took place at 2042 UT, 29 October as an X10/2B flare in AR0486 at S15W02. Since the model is a 1.5-D MHD model, we will implicitly assume that all four flares will be mimicked, as described below, at central meridian along the Sun-Earth line (unlike the 3-D kinematic procedure discussed by Dryer et al. [2004], who explicitly used the locations just described). The time delay, $\Delta t$, of each flare's start time for the simulation, starting from the first flare, is given in the last column of Table 1.

\subsection{Advanced Composition Explorer (ACE) Observations}

[10] Figure 2 shows ACE/SWEPAM/SWICS/MAG solar wind plasma and magnetic field data between 28 October and 1 November 2003. The bottom panel shows the solar wind velocity profile. Three shocks (marked by the vertical dotted lines) occurred at DOY 301.06, 302.24, and 303.68 (2003). A discontinuity, marked DD (to be discussed later), is noted by a vertical solid line at a smaller velocity increase from $\sim 600 \mathrm{~km} / \mathrm{s}$ to $650 \mathrm{~km} / \mathrm{s}$ at DOY 301.37, 2003. The proton density, in the second panel from the bottom, is not available [Skoug et al., 2004] in a reliable form during the period from $0600 \mathrm{UT}, 29$ October, to $0400 \mathrm{UT}, 30$ October 2003. Veselovsky et al. [2004, Figure 7], however, noting this problem, suggest that simulated density and velocity profiles obtained during the near real time project described by Dryer et al. [2004] are realistically satisfactory. However, we use the ACE data estimated by Skoug et al. [2004] and Zurbuchen et al. [2004]. The third panel for the temperature is notable for the flare-like value of $\mathrm{T}=10^{7}{ }^{\circ} \mathrm{K}$ reached immediately after the second shock's arrival. The next two panels, moving upward in Figure 2, show the total IMF magnitude and its three components: $\mathrm{Bx}, \mathrm{By}$, and $\mathrm{Bz}$ in the GSE (geocentric solar ecliptic) coordinate system. The next two panels near the top give the three velocity components (Vx, Vy, and Vz) with gaps behind the second shock as discussed by Skoug et al. [2004]. The top panel, which repeats the Dst response of the magnetospheric ring current, shows the two observed sudden storm commencements (SSCs), marked by the vertical dashed lines.

[11] As mentioned earlier, we identified in Table 1 the four flares that we believe are associated with the solar wind plasma velocity increases. An LDE M1.7/SF flare (25 October, $0552 \mathrm{UT}$, in AR0484 at N00W15) is related to the first velocity increase from $500 \mathrm{~km} / \mathrm{s}$ to $600 \mathrm{~km} / \mathrm{s}$ on 27 October. Another LDE X1.2/3N flare (26 October, 0617 UT, in AR0486 at S18E33) generated a directional discontinuity (after a shock interaction to be discussed below in greater detail) with a velocity jump on 28 October from $600 \mathrm{~km} / \mathrm{s}$ to $800 \mathrm{~km} / \mathrm{s}$. An X17/4B flare (28 October, 1102 UT, in AR0486 at S18E08) generated a shock on 29 October with an observed velocity jump from $800 \mathrm{~km} /$ $\mathrm{s}$ to more than $1600 \mathrm{~km} / \mathrm{s}$. Finally, in this subset of flares, an X10/2B flare (29 October, 2042 UT, again in AR0486 at S15W02) generated a shock on 30 October with a velocity jump from $900 \mathrm{~km} / \mathrm{s}$ to $1500 \mathrm{~km} / \mathrm{s}$. These three shocks and discontinuity (DD) are indicated in Figure 2 by three vertical dashed lines and a single solid line, respectively. Our primary objective is to trace the evolution of these shocks from the Sun, to indicate their interactions and products, and to compare them with the observed shocks and velocity profiles.

\subsection{Transit Time From Sun to Earth (L1)}

[12] Table 2 shows the occurrence and time delays between the four chosen flares (observed by GOES-12/ SXI) and shocks that were observed at ACE. The time delay, $\Delta T$, represents the elapsed time between the flare (as defined in the footnote of Table 1) and the corresponding shock observed at L1. The time delay, $\delta t$, represents the elapsed time between the flare under discussion and the previous flare. We suggest that the first two of the original flare-driven shocks experienced an interaction that resulted in a single shock plus a directional discontinuity (Figure 2 and section 5.1).

\section{The 1.5-D MHD Simulation Results}

\subsection{Choice of Pressure Pulse}

[13] We first performed a parametric study using the adaptive grid 1.5-D MHD code to obtain temporal solar wind velocity profiles at 1 AU by using only velocity, temperature, and density pulses. We did not consider magnetic flux emergence in these 1.5-D exercises. After testing various input combinations with various amplitudes and temporal durations, we concluded that only a combi- 

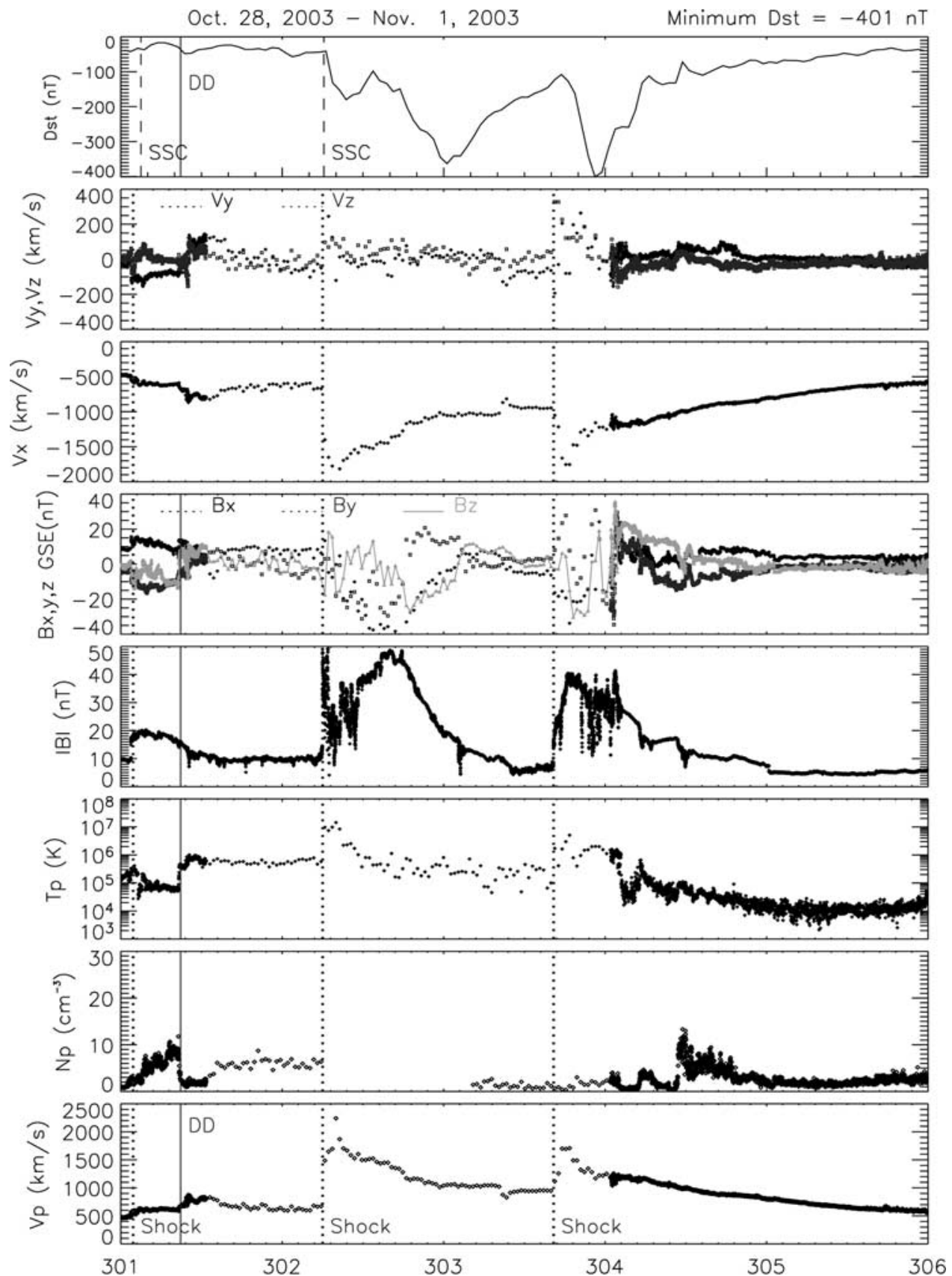

Figure 2. Solar wind plasma and magnetic field data and Dst index during 28 October and 1 November 2003. See color version of this figure in the HTML.

nation of temperature and density (i.e., a pressure pulse) could simulate appropriate shock and other discontinuity observations. (Our simulation results (not shown) demonstrated that the velocity and density, separately and combined, were unable to produce a shock wave with immediate postshock solar wind plasma speeds greater than $1000 \mathrm{~km} / \mathrm{s}$ near the Sun to mimic the observed LASCO CMEs for Halloween 2003 events.) In general, as noted by Smith and Dryer [1990] in 2-D MHD simulations, the ability to generate shocks is fundamentally a function of the total energy liberated by a particular solar event regardless of whether that event is a solar flare or helmet streamer eruption, etc.
Table 2. Flares and Associated Shocks Observed at $1 \mathrm{AU}$

\begin{tabular}{cccc}
\hline Flare & Shock & $\Delta \mathrm{T}^{\mathrm{a}}$ & $\delta \mathrm{t}^{\mathrm{b}}$ \\
\hline $0522 \mathrm{UT}$, & $0126 \mathrm{UT}$, & 68 hours $28 \mathrm{~min}$ & \\
$2003-10-25$ & $2003-10-28^{\mathrm{c}}$ & & \\
$0617 \mathrm{UT}$, & $0150 \mathrm{UT}$, & 43 hours $33 \mathrm{~min}$ & 24 hours 55 min \\
$2003-10-26$ & $2003-10-28$ & & \\
$1102 \mathrm{UT}$, & $0600 \mathrm{UT}$, & 18 hours $58 \mathrm{~min}$ & 52 hours $35 \mathrm{~min}$ \\
$2003-10-28$ & $2003-10-29$ & & \\
$2041 \mathrm{UT}$, & $1620 \mathrm{UT}$, & 19 hours $38 \mathrm{~min}$ & 33 hours 40 min \\
$2003-10-29$ & $2003-10-30$ & & \\
\hline
\end{tabular}

${ }^{\mathrm{a}}$ Here $\Delta \mathrm{T}$ is time delay between flare and shock observed at $1 \mathrm{AU}$.

${ }^{\mathrm{b}} \mathrm{Here} \delta \mathrm{t}$ is time delay between flares.

${ }^{c}$ Actually, this time marks the arrival of the discontinuity, DD, as discussed in the text as a product of the interaction of S1 and S2 (see section 5). 


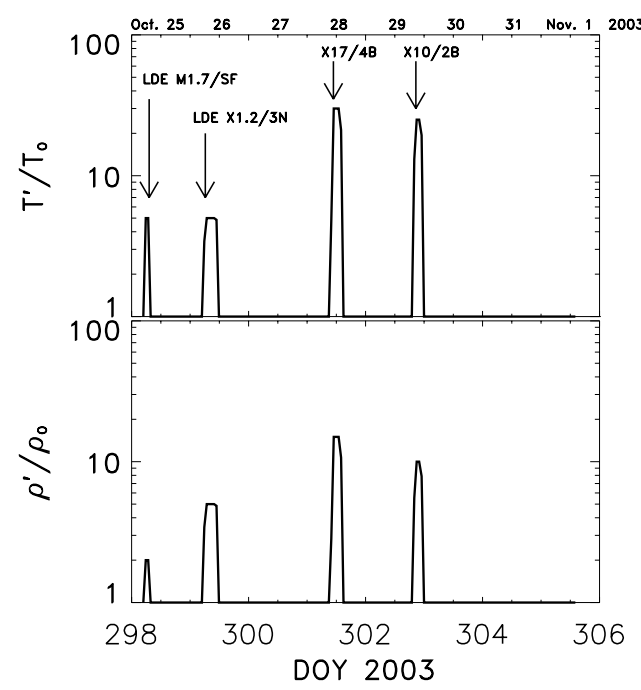

Figure 3. Evolution of perturbation variables at the lower boundary where $\rho \circ$ and $T \circ$ are the steady state values of density and temperature; $\rho^{\prime}$ and $T^{\prime}$ represent the jump of density and temperature relative to the steady state. The pressure pulses represent proxies for the indicated solar flares on the indicated dates. The first, second, third, and fourth pulses are initiated at $t=0,24.42,77.0$, and 110.67 hours, corresponding to the time delays between the four indicated "flares" (see also Table 1).

[14] First, we added a single pressure pulse with different magnitudes and temporal profiles to mimic, separately, the four separate flares as given in Table 1. The purpose was to achieve the actual shock arrival times at ACE. The choice of pressure pulses for mimicking the observed flares is appropriate for this study. For example, the observational study of Sheeley et al. [2000] shows that shocks appeared at the leading edges of halo CMEs that followed some flares. Furthermore, in 2.5-D MHD simulations of different physical mechanisms for CME accelerations, S. T. Wu et al. [2004] demonstrated that additional heating (i.e., a pressure pulse) could "simulate an active region flux rope accompanied by a flare to launch a CME." We are not considered in this paper with helmet-streamer-destabilized CMEs that can trigger solar flares. The latter topic is beyond the scope of this study. For each event, we chose the case with best results that reasonably matched the observations in both shock arrival times and the immediate postshock solar wind plasma speeds at L1. We then added four pressure pulses at successive times (the $\Delta t$ given in Table 1). We used an iterative procedure to adjust the input parameters for pulses $2-4$ to obtain the observed shock arrival times. The procedure is described in more detail, together with our results, in section 4.2.

\subsection{Simulation Results}

[15] Our previous simulations [Wu et al., 2005b] showed, as expected, that the shock arrival times at Earth depend on the magnitudes of the background solar wind speeds, particularly in the disturbed, postshock region. During the time-dependent evolution of the mimicked flare responses, the upstream propagations of fast mode MHD waves from pressure pulses 2,3 , and 4 are not steady-state as they move in the wake generated by pressure pulse 1 . This fact is also obvious for pressure pulses 3 and 4 as they propagate in the wake generated by pressure pulses 1 and 2 , and so on. Therefore we had to adjust, via trial and error, the sizes of pressure pulses 2,3 , and 4 , as obtained in the initial procedure described in section 4.1 , in order to achieve the observed shock arrival times at ACE. This procedure required many iterations to choose a final combination of pressure pulses to satisfy this goal. We consider the final choices (Figure 3 ) to be physically reasonable but make no claim that these combinations are unique.

[16] We classify the perturbations with various combinations of physical parameters: $\left(\frac{\rho^{\prime}}{\rho_{\mathrm{o}}} ; \frac{T^{\prime}}{T_{\mathrm{o}}}\right) . T_{\mathrm{o}}$ and $\rho_{\mathrm{o}}$ represent the "steady state" of the solar wind. $T^{\prime}$ and $\rho^{\prime}$ represent the variations of temperature and density, respectively, that are assumed, as just discussed, to mimic each flare. The temporal variation of the perturbation is a "near square wave" with a 1-hour rise and decay separated by various constant magnitude durations at $1 R_{\odot}$. Four pressure pulses (Figure 3) were chosen after the trial and error procedure described above. The sequential pressure pulses were initiated at time, $\mathrm{t}=0,24.42,77$, and 110.67 hours that correspond to the times (in DOY, 2003) 298.24, 299.26, 301.45 , and 302.86 , respectively. The launch time of each pressure pulse, then, is identical with the flare onset time on the Sun as noted in Tables 1 and 2. Figure 3 shows the temporal evolution of the four pressure pulses. For example, the largest pressure pulse (third one) represents the largest, X17/4B, flare. Pulse 1, then, will propagate into the steady state solar wind plasma and magnetic field shown in Figure 4 from the Sun to $400 R_{\odot}$. The vertical line indicates the location of $1 \mathrm{AU}$.

[17] Figures $5 \mathrm{a}-5 \mathrm{~d}$ show the simulation results of the solar wind solution versus heliocentric distance at $\mathrm{t}=70.1$,

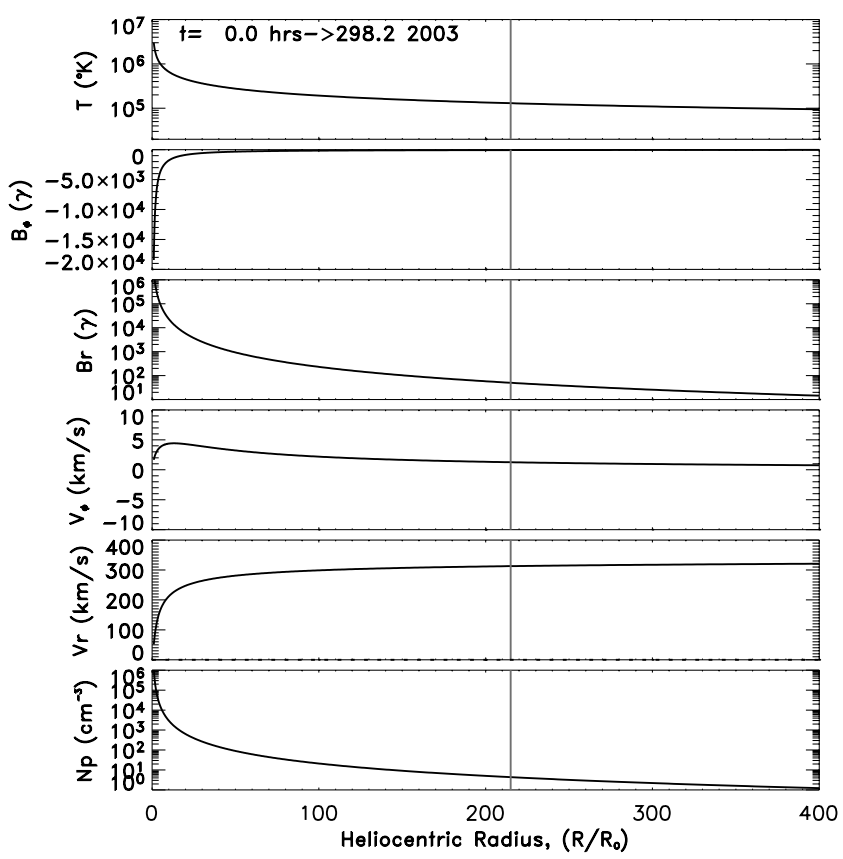

Figure 4. The initial steady state solar wind solution for the present 1.5-dimensional (1.5-D) MHD study. Earth's location at $1 \mathrm{AU}$ is indicated by the solid vertical line. See color version of this figure in the HTML. 

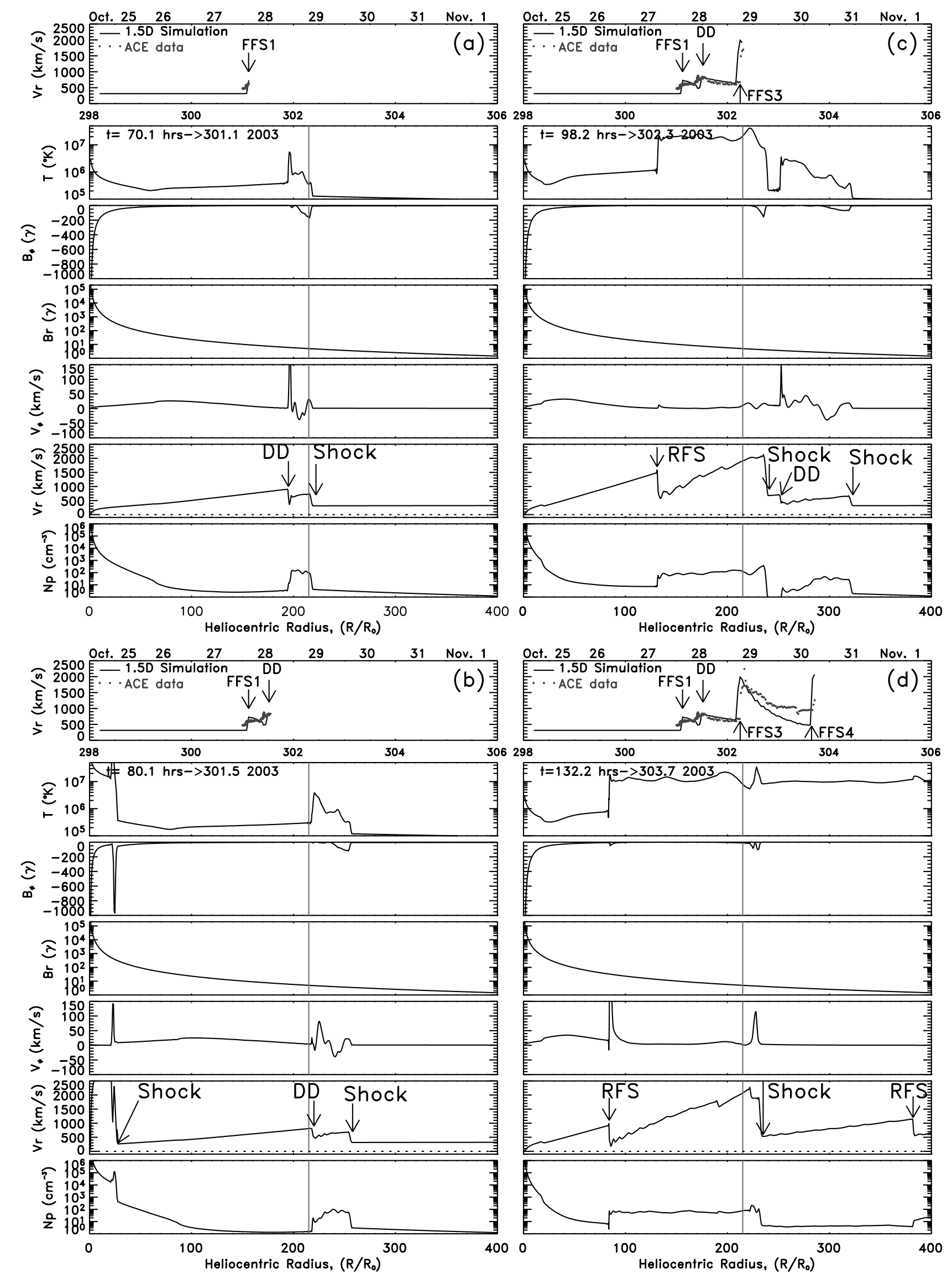

Figure 5 


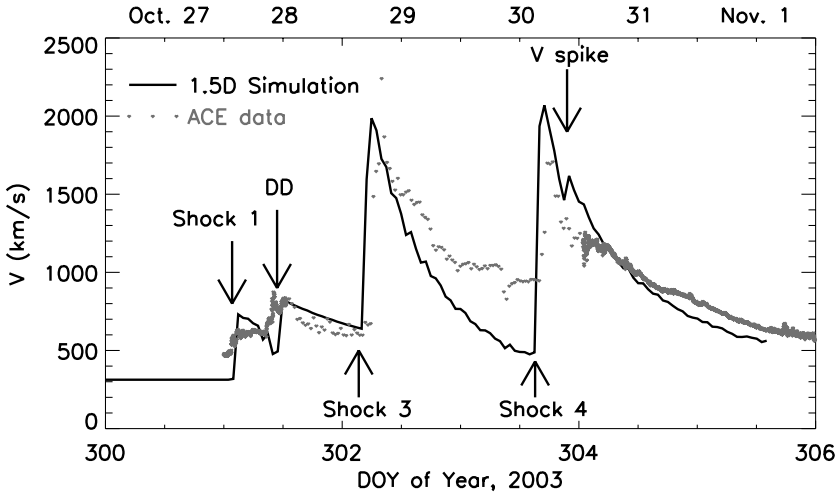

Figure 6. Simulation (solid line) and observed (dotted line) solar wind plasma speed. Continuation of the uppermost panels in Figure 5 until the end of the simulation. Excellent agreement with the observations is shown for the simulated shock times of arrival at ACE as well as the general level and amplitude of the speed profile. The "V spike", following Shock 4, is a reverse shock; see, also, "RS" in Figure 9's helioradial-time diagram. See color version of this figure in the HTML.

$80.1,98.2$, and 132.2 hours, respectively. The top panel in each quadrant (marked Figure 5a to 5d) of the figure shows the evolution of solar wind speed that is monitored at L1. The solid lines represent the simulation results, and the dotted lines show the observation by ACE. At $\mathrm{t}=70.1$ hours, DOY 301.1 (28 October 2003), one shock arrived at L1, and a discontinuity (DD) is approaching that location as shown in the lower six panels of Figure 5a. The discontinuity, DD, might possibly be assumed to be a reverse shock because it is marked by a sudden speed increase accompanied by a density and temperature decrease. We will comment further on this point below. As discussed earlier, the top panel of Figure 5a shows that Shock 1 (a forward fast shock, hereafter, FFS1, etc.) arrived at L1 in both the simulation and observations.

[18] At $\mathrm{t}=80.1$ hours, DOY 301.5 (28 October 2003), bottom left side of Figure $5 \mathrm{~b}$ (all seven panels), the DD just passed $1 \mathrm{AU}\left(=215 R_{\odot}\right)$, and a second shock (discussed in section 5.1) is seen at $28 R_{\odot}$. Once again, however, DD is likely to be assumed, erroneously, to be a reverse shock that follows its "partner," FFS1. We will show in section 5.1 that a much more complicated interaction process has taken place. ACE's observation clearly shows that DD has just passed that location. At $\mathrm{t}=$ 98.2 hours, (DOY 302.3, 29 October 2003), in Figure 5c, the third shock (FFS3) just passed L1, and a reverse fast shock (RFS) was clearly formed and located at $131 R_{\odot}$. Figure $5 \mathrm{~d}$, at $\mathrm{t}=132.2$ hours (DOY 303.7, 30 October 2003), shows that the fourth shock (FFS4) just passed L1, and another reverse shock (RFS) had been formed and, at this time, is located at $84 R_{\odot}$.

[19] Figure 6 shows, in higher resolution, the solar wind speed monitored at ACE in both the simulation (solid line) and observations (dotted line). The arrival times of the three simulated shocks (FFS1, FFS3, and FFS4) and the discontinuity (DD) match quite well with ACE's observations. In addition, the simulated plasma speed profiles behind the three shocks are also in good agreement with the observations. The reader is reminded that the simulated shock arrival times were already matched to the actual arrival times via the trial and error procedure discussed earlier. The DD comparison, however, is considered to be an important new result. Also, the good agreement of the velocity profiles suggests that this pressure pulse procedure, albeit nonunique, is physically realistic as a flare proxy as discussed in section 4.1 .

\section{Discussion}

[20] C.-C. Wu et al. [1996b, 2004, 2005a] showed (in intensive parametric studies) that a positive, square-wave perturbation (pressure pulse), for example, will generate both fast and slow shocks. In the present more complicated but realistic simulation, we added four perturbations to mimic the effect due to flares at the Sun, but only three fast shocks were observed at L1. The following section will discuss this issue in greater detail because of its overall complexity that may easily arise in other cases.

\subsection{Shock-Shock Interaction Within 1 AU}

[21] Figures 7 and 8 show the process of shock-shock interaction between shocks created by both pressure pulses 1 and 2 in higher spatial resolution. Thus Figure 7 shows the simulated plasma parameters and magnetic field $\left(B_{\phi}\right.$ only since $B_{r}$ is always constant across any discontinuity in 1.5-D MHD calculations due to magnetic flux conservation). The four quadrants, Figures $7 \mathrm{a}$ to $7 \mathrm{~d}$, show these physical parameters, respectively, from $t=24$ hours (DOY 299.2) until $t=60.1$ hours (DOY 300.7). Figure 8 is a continuation of Figure 7 and extends these simulated profiles in Figures $8 \mathrm{a}-8 \mathrm{~d}$, respectively, from $\mathrm{t}=63.1$ hours (DOY 300.8) until $\mathrm{t}=76.1$ hours (DOY 301.4). (Note that the last quadrant, Figure 8d, fits, temporally, between Figures 5a and 5b.) At $t=24$ hours (Figure 7a), before the second pressure pulse was launched, a forward fast shock (FFS1) and a reverse slow shock (RSS1) were generated. During this time, a slow shock-like structure (SSL) formed

Figure 5. The dynamic solar wind solution versus heliocentric distance for $\mathrm{t}=70.1,80.1,98.2$, and 132.2 hours, respectively, as described as follows: (a) $\mathrm{t}=70.1 \mathrm{hr}$ (upper left quadrant of figure). The uppermost panel shows the solar wind speed monitored at L1. The solid line represents the simulation result, and the dotted lines show the observations by the ACE spacecraft at 70.1 hours (simulation time). The six lower panels in this quadrant show, at this time, temperature, T; azimuthal magnetic field component, $B_{\phi}$; radial magnetic field component, $B_{r}$; azimuthal velocity component, $V_{\phi}$; radial velocity, $V_{r}$; and proton number density, $\mathrm{Np}$, respectively. (b) $\mathrm{t}=80.1$ hours (lower left quadrant). Progression in time from Figure 5a; (c) $\mathrm{t}=98.2$ hours (upper right quadrant). Continued, "animated" progress in time from Figure 5b; and $(\mathrm{d}) \mathrm{t}=$ 132.2. hours (lower right quadrant). Continued temporal progression. The words indicated by the arrows (Shock, DD, etc.) are discussed in the text. See color version of this figure in the HTML. 

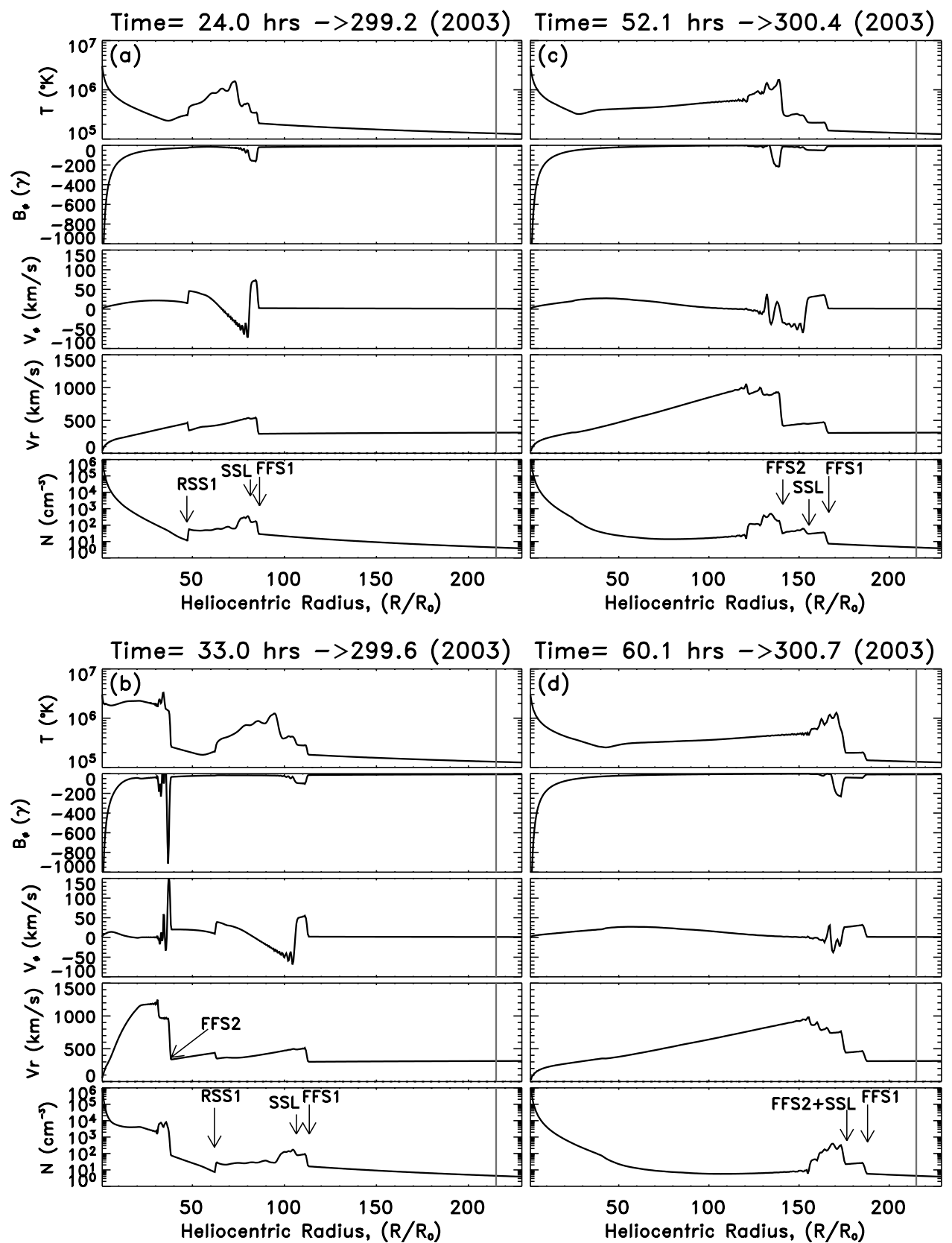

Figure 7. Solar wind plasma and magnetic field data for time $=24,33,52.1$, and 60.1 hours, respectively. The vertical line indicates Earth's location. The sequence of discontinuity interactions is described in the text. See color version of this figure in the HTML.

from internally interacting compression and rarefaction waves between FFS1 and RSS1. At $\mathrm{t}=33$ hours (Figure 7b, lower left quadrant of Figure 7), the second pressure pulse created a forward fast shock (FFS2) that propagated behind RSS1. At $\mathrm{t}=52.1$ hours, the FFS2 interacted with and passed through RSS1 and was propagating behind SSL (see Figure 7c). At $\mathrm{t}=60.1$ hours, FFS2 overtakes SSL from behind (see Figure 7d) and separates from SSL later (see Figure $8 \mathrm{a}$, at $\mathrm{t}=63.1$ hours). Then, FFS2 overtakes FFS1 from behind, with the interaction product becoming a new shock, FFS1 +2 (see Figures 8b, 8c, and 8d). During that period, the SSL was interacting with the ambient solar wind and formed a discontinuity, DD, called because of a temporarily induced cross shear, $V_{\phi}$ The vertical lines indicate the location of $1 \mathrm{AU}$ in this case just prior to DD's arrival. This DD is generated by the time-dependent interaction of the nonuniform solar wind with the temporally varying and propagating shock and following flows. It is in a transition condition and is very unstable, as shown in Figures $8 b-8 d$. Further investigation (beyond the scope of the present study) is required to learn the real characteristics of the DD. Therefore we call it as a discontinuity (DD) in this study. It is clear that the simulated density was decreasing, the temperature was increasing, and the velocity was slightly fluctuating. During this same period, ACE observed this similar phenomenon as 

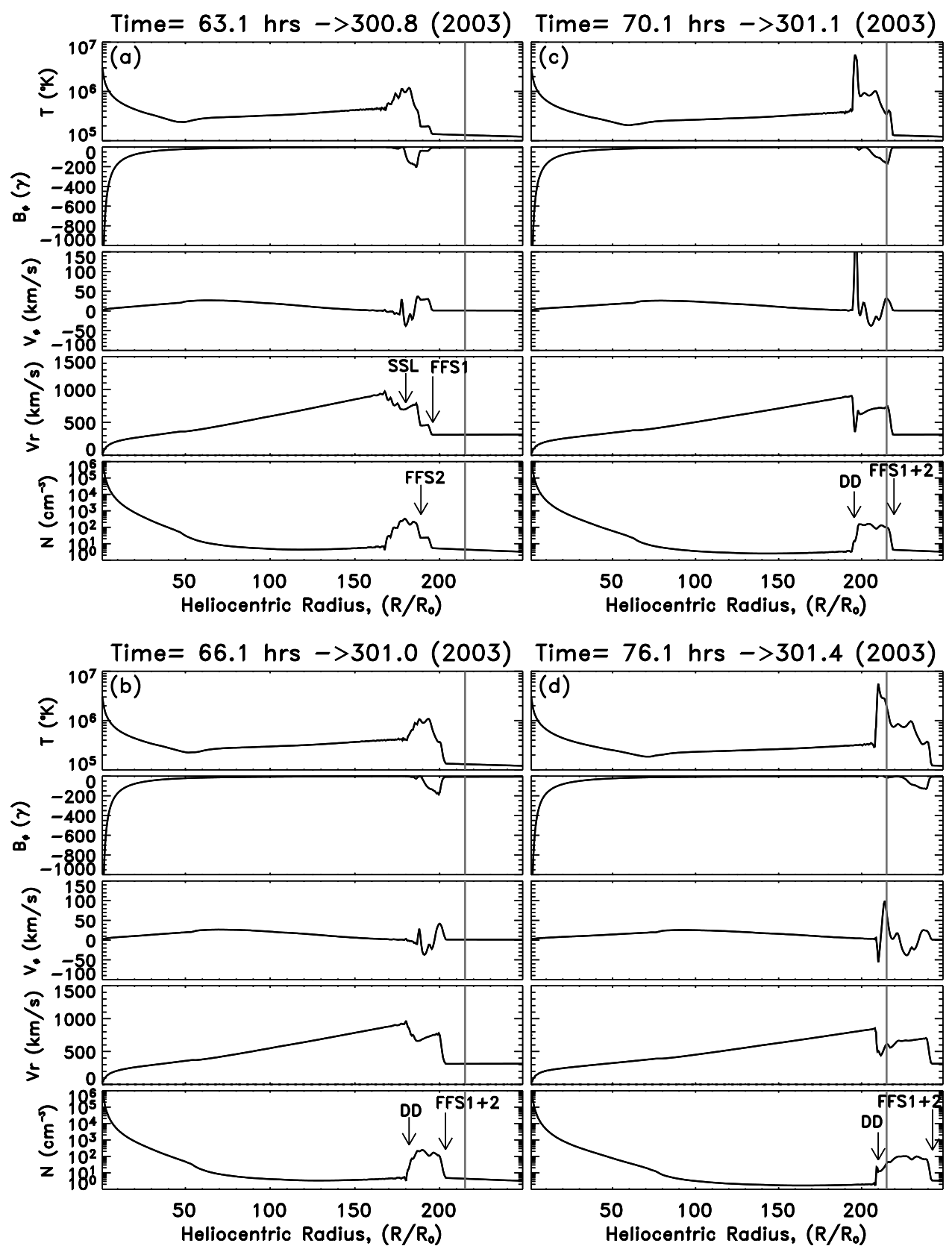

Figure 8. Solar wind plasma and magnetic field data for time $=63.1,66.1,70.1$, and 76.1 hours, respectively. The vertical line indicates Earth's location. The sequence of discontinuity interactions is described in the text. See color version of this figure in the HTML.

shown in Figure 2. Thus the discontinuity that might have been taken to be a reverse shock, as discussed earlier, is now found, via the simulation, to be a directional discontinuity and not a RFS at all!

[22] Figure 9 shows the helioradius-time profiles of the simulation results. The solid black lines indicate the evolution of the shock waves and other discontinuities. In Figure 9, DD means discontinuity and RFS means reverse fast shock. FFS1, FFS2, FFS3, and FFS4 refer to the forward fast shocks created originally by the pressure pulses $1,2,3$, and 4 , respectively. The figure shows that FFS1 was overtaken by FFS2 from behind and became a new shock (FFS1 + FFS2). From the slopes (i.e., speeds) of FFS1, FFS2, and FFS1 + FFS2, it is clear that the strength of the new shock is stronger than that of FFS1 but is weaker that it was for FFS2. The additional product of this interaction, DD, was swept away after shock FFS3 overtakes it. Shock FFS3's original reverse shock, RFS3, is shown to be very strongly amplified after FFS4 overtakes it. FFS4's original reverse shock partner (RFS4, in the lower right portion of Figure 9) appears to resist its outward convection by the solar wind at first but finally, losing strength, is once again convected outwardly. We suspect that it probably decays to a fast mode MHD wave well within $1 \mathrm{AU}$.

[23] Figure 10 shows a stack plot of the comparative temporal evolution of the solar wind velocity in the radial direction during the entire simulated procedure. The numbers on the right side of Figure 10 show the time of the 


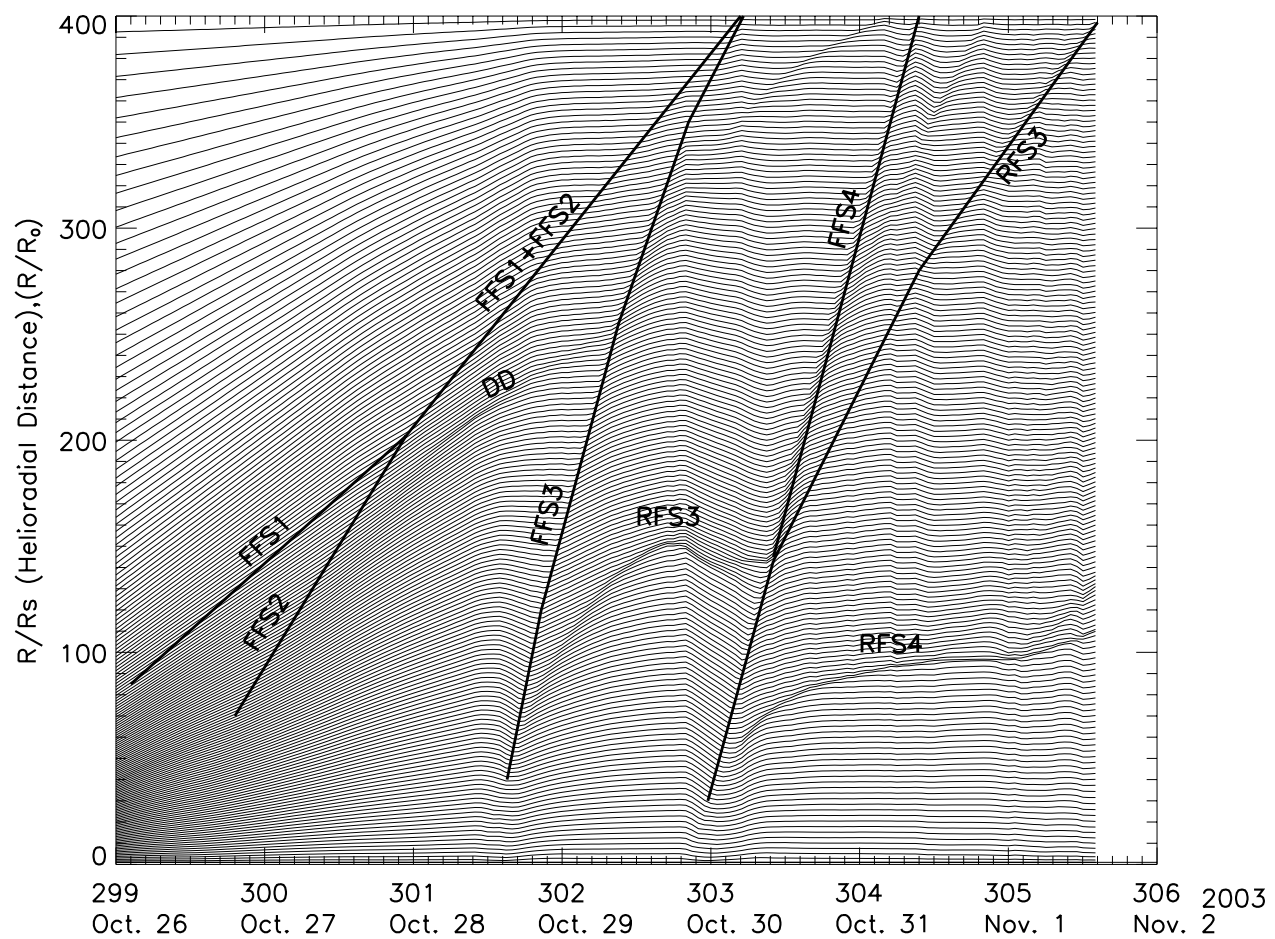

Figure 9. Helioradial-time diagram of the various shocks, their products after interaction, and the adaptive grid spacings.

simulation (e.g., $\mathrm{t}=0$ hours represents DOY 298.2, 0552 UT, 25 October 2003) when the first pressure pulse was launched at the Sun. The time resolution is 3 hours for the ascending scale that is also marked by the arrows for the first pressure pulse (PP), etc., at the appropriate times given in Tables 1 and 2. The velocity scale on the left side of Figure 10 relates to the first PP. The scale is then sequentially moved upward for each time step shown. The dotted lines represent the various shock trajectories. Again, this plot shows that FFS1 and FFS2 merged into a new shock before either one reached 1 AU. The propagating shock speeds are not constant when the plasma speed upstream of each shock is not constant; note, in particular, the early stages of FFS2, FFS3, and FFS4. The effect of this upstream inhomogeneity is dramatically illustrated in the 3-D kinematic studies [e.g., Dryer et al., 2004] that demonstrated global shock shape warping in the ecliptic plane.

\subsection{Comparison Between Simulation and Observation: A Remark}

[24] A halo CME was first observed by SOHO/LASCO at 1130:05 on 28 October 2003. The estimated speed of the leading edge from 2 to $32 R_{\odot}$ is $\sim 2459 \mathrm{~km} / \mathrm{s}$ [Gopalswamy et al., 2005]. From the simulation results, we calculated the speed of the shock wave that is related to this third flare (X17/4B, 1102 UT, 28 October 2003) by using the Wave Transit Method (WTM). The detailed computational procedure for WTM is given by $W u$ et al. [1996b]. We found that the shock speed increased from $1800 \mathrm{~km} / \mathrm{s}$ (at $4 R_{\odot}$ ) to $2400 \mathrm{~km} / \mathrm{s}\left(60 R_{\odot}\right)$ with an average speed of $\sim 2100 \mathrm{~km} / \mathrm{s}$. This average shock speed is close to the halo CME's speed that was derived from the coronagraph observations of $\mathrm{SOHO} / \mathrm{LASCO}$. We might suggest, as a result of this comparison, that the CME's leading edge was actually the shock itself followed by strong compression of the coronal density and associated Thomson scattering from the compressed electrons.

\subsection{Solar Sources for Geomagnetic Storms: Another Remark}

[25] We have already mentioned in section 1 the various statistics of the solar flares and geomagnetic storms that rank among the largest of the solar cycle 23. It is clear that it is not productive to discuss, for physical purposes, the "size" of a flare (in terms of its soft X-ray classification) and the "size" of the ensuing geomagnetic storm (in terms of, say, the Dst index). Such comparisons as noted in section 1 can be misleading. Rather, we remark that the more important scientific objective is to provide the interplanetary linkage between the suspected solar event, large or small, and the geomagnetic consequence, again large or small. We return to the present simulation as a case in point.

[26] The simulation results show that both the first and second pressure pulses generated their own shocks. We have seen how both shocks merged into a single shock (FFS1 + FFS2) with the creation of DD before propagating to 1 AU. The shock observed by ACE on 28 October 2003, $0150 \mathrm{UT}$, was created by FFS1 + FFS2 that also is related to an SSC at 0206 UT on 28 October. The Dst index dropped to $-48 \mathrm{nT}$ for this geomagnetic storm.

[27] The third pressure pulse (or "flare") was stronger than the fourth one. Each case generated very fast shocks (Vs $\sim 2000 \mathrm{~km} / \mathrm{s}$ ) that took less than a day to arrive at $1 \mathrm{AU}$. The shock generated by the third pulse was the faster one, but it did not create the most intensive storm during the study period because it did not have sufficient time for the magnetospheric processes to be completed [Veselovsky et al., 2004]. That is, the shock generated by the fourth pulse 


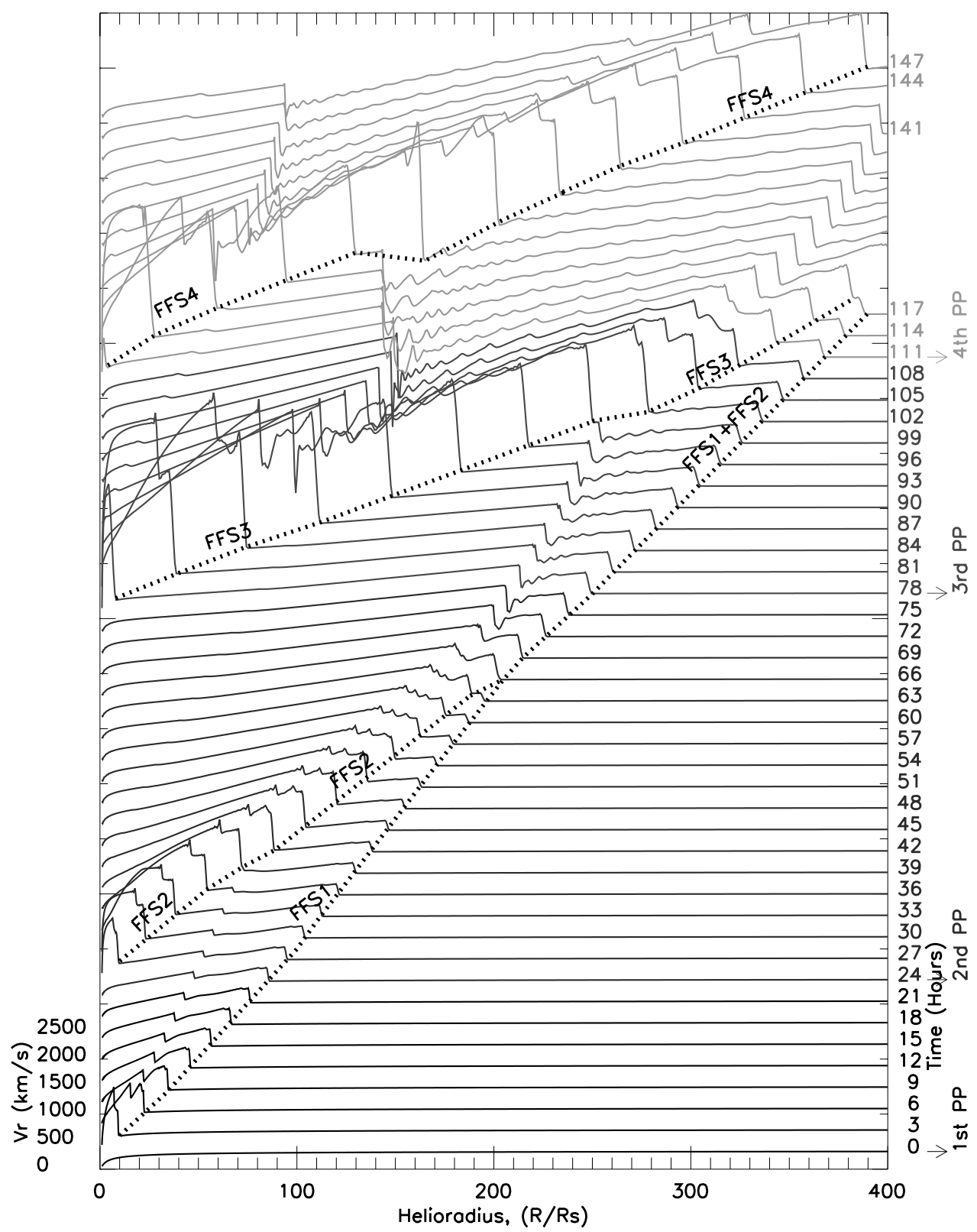

Figure 10. Solar wind speed as a function of helioradius for the 1.5-D MHD simulation of interacting shocks and other discontinuities. The velocity scale on the left side corresponds to the time, $\mathrm{t}=0$ hours which is indicated on the right side of the figure when the first pulse (first PP) is initiated. This scale is then moved up, when the initiation of the second PP is initiated, so that $V_{r}=0$ will coincide with the second, marked arrow. This process is repeated for the third PP and fourth PP. The color velocity profiles (black, blue, red, and orange) correspond, respectively, to the four pulses that mimic each of the flares in Tables 1 and 2. See color version of this figure in the HTML.

arrived while the Dst was descending with the subsequent "completion" of the substorm processes marked, finally, by the general ring current index $(-401 \mathrm{nT})$ as discussed in section 1. Thus we remark that it is unproductive, scientifically and operationally, to compare flare sizes and geomagnetic storm sizes via any of the standard classifications.

\section{Conclusions}

[28] Motivated by a subset of the "Halloween 2003 epoch" solar flares and geomagnetic storms, we used 1.5-D
MHD simulations initiated at the solar surface to study the shocks generated from each event in order to study their interactions and relative evolution. We used a trial and error procedure, using temperature and density pulses (i.e., pressure pulses), to choose a set that adequately produced the actual shock arrival times at ACE's L1 position. We found that the simulated solar wind velocity profile was, accordingly, in good agreement with the observations. Also of significance is the fact that the 1.5-D MHD simulation is well suited to study fundamental characteristics of an extremely fast shock (Vs $>$ $2000 \mathrm{~km} / \mathrm{s}$ ) propagation from the Sun to the Earth from 


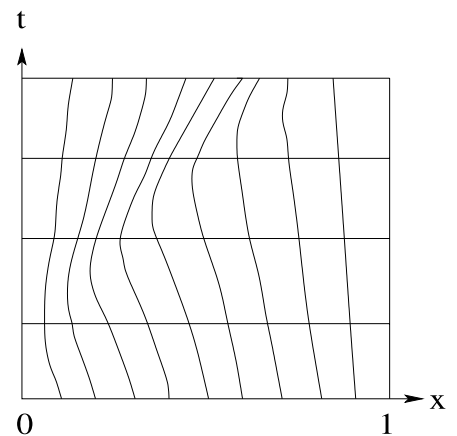

(a) Physical

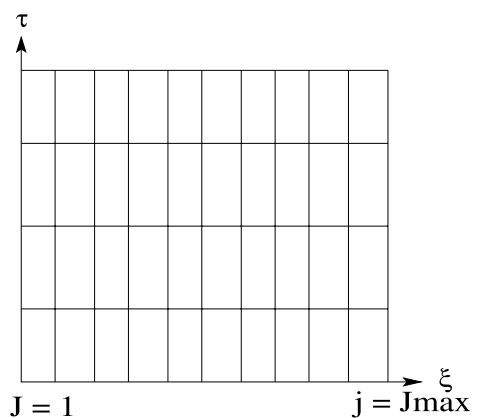

(b) Computational

Figure A1. One-dimensional coordinates (adapted from Panitchob [1987]).

the 28 October 2003 flare including the high in situ postshock plasma speed $(>2000 \mathrm{~km} / \mathrm{s})$. Our adaptive grid's MHD simulation also helped to understand why some solar disturbances did not generate shocks that survived at Earth. That is, we showed how shock interactions are an important process that should be considered for solar flare-geomagnetic storm associations (e.g., FFS1 and FFS2 combined to form FFS1 + FFS2 that generated SSC1). We conclude that interaction studies, such as the present one, are essential for the study of staccato solar events of any kind and their association with specific geomagnetic events.

\section{Appendix A}

\section{A1. Basic Theory}

[29] To our knowledge, the first application of an adaptive grid finite difference technique (MHD model) in the field of astrogeophysics is made by Panitchob [1987] and Panitchob et al. [1987], who used an adaptive grid method in the simulations of MHD flows in the solar corona and the solar wind. The adaptive grid model given by Panitchob [1987] which is described in detail as follows.

[30] For the solution of finite difference equations, the data are typically stored in ordered arrays of points. The ultimate goal is how to assemble these points in such a way that the solution of the difference equations is optimal. The problem associated with both grid generation and grid adaptations is to establish the mapping relationship between physical space and computational space. In the computational space, the points are uniformly distributed. In the physical space, the points may adjust their positions to follow gradients developing in the evolving physical solution (see Figure A1). Since the physical solution is not known a priori, the only way to approach an optimum grid is to adapt the grid to the solution in a time-like manner. For simplicity of illustration, consider the one-dimensional transformation to computational space where

$$
\begin{aligned}
& t \rightarrow \tau \\
& x \rightarrow \xi
\end{aligned}
$$

The time rate of change of the dependent variable $u$ then becomes

$$
\frac{\partial u}{\partial t} \rightarrow \frac{\partial u}{\partial \tau}+\frac{\partial \xi}{\partial t} \frac{\partial u}{\partial \xi}
$$

where $\frac{\partial \xi}{\partial t}$ is the grid speed which must be either specified or determined numerically. A convenient method for approximating the grid speed numerically has been to use backward first-order differences in time.

[31] In the model developed by Panitchob [1987], the numerical grid generation technique is based on a variational principle. The method was put forward by Winslow [1966]. Winslow formulates the zoning problem as a potential problem with the mesh lines playing the role of equipotentials. This model is to use the variational approach to generate the adaptive grids and then apply them to a class of MHD flow problems. For one-dimensional adaption by using the variational approach, the total error is reduced when the grid points are distributed as,

$$
\int_{x_{i}}^{x_{i+1}} w(x) d x=\text { const }
$$

or, in discrete form

$$
\left(\Delta x_{i}\right) w_{i}=\text { const }
$$

for all $i$, where $w(x)$ is a positive weighting function.

[32] The nonuniform point distribution can be considered as a transformation, $x(\xi)$, from a uniform grid in $\xi$-space, with the coordinate $\xi$ serving to identify the grid points. Then, equation (A5) can be represented as

$$
x_{\xi} w(\xi)=\text { const }
$$

where $x_{\xi}$ is the metric coefficient and corresponds to the ratio of arc lengths in physical and computational space. Equation (A6) is just the Euler-Lagrange equation for the minimization of the integral

$$
I=\int_{0}^{1} w(\xi) x_{\xi}^{2} d \xi
$$


This integral can be taken to represent the energy of a system of springs with spring constants $w(\xi)$, spanning each grid interval assuming all the points to have been expanded from common points so that $x_{\xi}$ is the extension of the spring at $\xi$. The grid point distribution resulting from the equidistribution thus represents the equilibrium state of such a spring system. The constant in equation (A6) can be evaluated, and an expression for grid-point spacing (in physical space) can be obtained as follows.

[33] For maintaining a uniform grid in $\xi$-space (i.e., computational space), the constant in equation (A6) can be evaluated by normalizing $x$ to the interval $(0,1)$. If $\xi$ is normalized to $(0,1)$, then by integration of equation (A6),

$$
\text { const. }=\frac{1}{\int_{0}^{1} \frac{d \xi}{w(\xi)}}
$$

then equation (A6) becomes

$$
x_{\xi}=\frac{1}{w(\xi) \int_{0}^{1} \frac{d \xi}{w(\xi)}}
$$

and since $\xi_{x}=1 / x_{\xi}$, the transformation is then determined by

$$
\xi(x)=\left\{\int_{0}^{1} w[\xi(\bar{x})] d \bar{x}\right\}\left\{\int_{0}^{1} \frac{d \xi}{w(\xi)}\right\}
$$

thus

$$
\Delta \xi=\left\{\int_{x_{i}}^{x_{i+1}} w[\xi(x)] d x\right\}\left\{\int_{0}^{1} \frac{d \xi}{w(\xi)}\right\}
$$

so that equation (A6) is realized by taking equal increments in $\xi$. With $\Delta \xi=1 / N$, where $N+1$ is the total number of points on the line, we then have from equation (A9), for the point spacing, the expression,

$$
\Delta x_{i}=\frac{1}{w\left(\xi_{i}\right) N \int_{0}^{1} \frac{d \xi}{w(\xi)}}
$$

where $N$ is the total number of grid intervals.

\section{A2. Adaptive Grid Scheme}

[34] In order to construct a moving grid in the physical space $(x, t)$; see Figure A1a, we consider a mapping to a computational space $(\xi, \tau)$ in which the grid lines are equally spaced and $\tau$-independent; see Figure A1b. The grid will be generated by numerically determining the transformation at time $(t+\Delta T)$ from the transformation at time $\mathrm{t}$ and the solution at time $\mathrm{t}$.

$$
\begin{gathered}
\xi=\xi(x, t) \\
\tau=t
\end{gathered}
$$

[35] Following the variational formulation described by Brackbill and Saltzman [1982], we seek to minimize Bell and Shubin [1983]:

$$
I=\int_{0}^{1}\left[x_{\xi}^{2}+\lambda_{a} w(\xi) x_{\xi}^{2}+\lambda_{b}\left(x_{\xi}-x_{\xi}^{*}\right)^{2}\right] d \xi
$$

where $\lambda_{a}$ and $\lambda_{b}$ are parameters, $w(\xi)$ is a weighing function scaled so that $0 \leq w(\xi) \leq 1$, and the asterisk symbol indicates the value of $x_{\xi}$ from the previous time step. To understand equation (A15), we may think of $x_{\xi}$ as $\Delta x / \Delta \xi$. The first term of equation (A15) attempts to equidistribute the grid size $\Delta x$ since the minimum value of this term is obtained for a uniform grid. The second term attempts to make $\Delta x$ small where the weighing function $w(\xi)$ is large, and vice versa. The third term is expressed in practice as a time difference and serves to restrict the movement of the grid. The choice of $\lambda_{a}$ and $\lambda_{b}$ determines the relative emphasis given to these effects.

[36] In order to numerically determine $x(\xi)$, we first find the Euler-Lagrange equation for the minimization of equation (A15). From the calculus of variations, the function $x(\xi)$ for which the integral $\int F\left(\xi, x, x_{\xi}\right) d \xi$ (i.e., equation (A15)) is an extremum, is given by the differential equation,

$$
\frac{d}{d \xi}\left[\frac{\partial F\left(\xi, x, x_{\xi}\right)}{\partial x_{\xi}}\right]-\frac{\partial F\left(\xi, x, x_{\xi}\right)}{\partial x}=0
$$

This equation is called the Euler-Lagrange equation of the variational problem.

[37] Then the Euler-Lagrange equation for the minimization of the integral (i.e., equation (A15)) becomes

$$
\frac{d}{d \xi}\left[2 x_{\xi}+2 \lambda_{a} w(\xi) x_{\xi}+2 \lambda_{b}\left(x_{\xi}-x_{\xi}^{*}\right)\right]=0
$$

or

$$
\left(1+\lambda_{a} w(\xi)+\lambda_{b}\right) x_{\xi \xi}+\lambda_{a} w_{\xi}(\xi) x_{\xi}-\lambda_{b} x_{\xi \xi}^{*}=0
$$

Replacing equation (A18) with a finite difference approximation and using the boundary conditions $x_{1}=0$ and $x_{J \max }=1$ yields a system of linear equations determining the new grid point locations at each time step.

[38] The form of the weighting function used here is

$$
w(\xi)=u_{\xi}
$$

where $u$ can be one of the solution variables (i.e., $v_{r}, v_{\theta}, v_{\phi}$, $B_{r}, B_{\theta}, B_{\phi}, \rho, p$, or T), or the combination of variables such as the total pressure, $\left(p+\left|B^{2}\right| / 8 \pi\right)$, which is characterized by the physics of the problem.

[39] From equation (A15), in the case of $\lambda_{b}=0$ (no grid smoothing in time), we obtain the Euler-Lagrange equation as

$$
\left(1+\lambda_{a} w\right) x_{\xi}=\text { const }
$$

so that the grids equidistribute $\left(1+\lambda_{a} w\right)$ in computational space, as expected. In the case of $\lambda_{a}=0$ (no clustering in space), we get the Euler-Lagrange equation as

$$
x_{\xi}+\lambda_{b}\left(x_{\xi}-x_{\xi}^{*}\right)=\text { const } \text {. }
$$

corresponding to a combination of smoothing in space and in time.

[40] The special effort we consider here is to vary the grid generation parameters $\lambda_{a}$ and $\lambda_{b}$. Choosing large values of 
$\lambda_{a}$ will result in a more accurate solution because grid points are more closely clustered at the shock fronts. However, such small grid spacing requires very small time steps unless $\lambda_{b}$ is chosen appropriately.

[41] Acknowledgments. This work is supported partially by the NASA Living with a Star program, NASA grant NAG-12527. CCW and DB are supported partially by NSF Space Weather Program, ATM-028414 DB thanks partial support with NASA-LWS grant NASW-02035. CDF and MD are supported by the University Partnering for Operational Support program (UPOS) of the DoD's USAF and US Army and also by the NASA's Living with a Star program via grant NAG5-12527 to Exploration Physics International, Inc. MD also acknowledges the hospitality of the NOAA Space Environment Center. STW wishes to acknowledge support of this work via National Science Foundation (NSF) grant ATM0310115 and NASA grant NAG5-12843. ZS and TRD acknowledge partially funding from NASA Living With a Star (LWS) Targeted Research and Technlogy program through NOAA Work Order W-10, 118. Work at Los Alamos was performed under the auspices of the U. S. Department of Energy, with financial support from the NASA ACE program. Finally, we thank W. Murtagh (Senior Forecaster, NOAA/Space Environment Center) for the information on large flare and ensuing geomagnetic storm classifications.

[42] Arthur Richmond thanks Thomas E. Holzer and Toshio Terasawa for their assistance in evaluating this paper.

\section{References}

Bell, J. B., and G. R. Shubin (1983), An adaptive grid finite difference method for conservation laws, J. Comput. Phys., 52, 569-591.

Brackbill, J. U., and J. S. Saltzman (1982), Adaptive zoning for singular problems in two dimensions, J. Comput. Phys., 46, 342-368.

Dryer, M., Z. Smith, C. D. Fry, W. Sun, C. S. Deehr, and S.-I. Akasofu (2004), Real-time shock arrival predictions during the "Halloween 2003 epoch," Space Weather, 2, S09001, doi:10.1029/2004SW000087.

Gold, T. (1955), Gas dynamics of cosmic clouds, in Gas Dynamics of Cosmic Clouds, edited by H. C. van de Hulst and J. M. Burgers, p. 103, Elsevier, New York.

Gopalswamy, N. A., S. Yashiro, Y. Liu, G. Michalek, A. Vourlidas, M. L. Kaiser, and R. A. Howard (2005), Coronal mass ejections and other extreme characteristics of the 2003 October-November solar eruptions, J. Geophys. Res., 110, A09S15, doi:10.1029/2004JA010958.

Hughes, W. F., and J. A. Brighton (1967), Fluid Dynamics, Schaum's Outline Ser., McGraw-Hill, New York.

Panitchob, S. (1987), An adaptive grid finite difference method for timedependent magnetohydrodynamic (MHD) flows and its astrogeophysical applications, Ph.D. dissertation, Dept. of Mech. Eng., Univ. of Ala., Huntsville, Ala.

Panitchob, S., S. T. Wu, and S. T. Suess (1987), An adaptive grid unsteady model for two-dimensional magnetohrodynamic (MHD) flow, Rep. AIAA-87-1218, Am. Inst. of Aeron. and Astron., Washington, D. C.

Priest, E. R. (1982), Solar Magnetohydrodynamics, Springer, New York

Sheeley, N. R. J., W. N. Hakala, and Y.-M. Wang (2000), Detection of coronal mass ejection associated shock waves in the outer corona, J. Geophys. Res., 105, 5081-5092.

Skoug, R. M., J. T. Gosling, J. T. Steinberg, D. J. McComas, C. W. Smith, N. F. Ness, Q. Hu, and L. F. Burlaga (2004), Extremely high speed solar wind: 29-30 October 2003, J. Geophys. Res., 109, A09102, doi:10.1029/ 2004JA010494

Smith, Z., and M. Dryer (1990), MHD study of temporal and spatial evolution of simulated interplanetary shocks in the ecliptic plane within $1 \mathrm{AU}$, Sol. Phys., 129, 387-405.
Smith, Z., M. Dryer, and S. M. Han (1986), Interplanetary shock collisions: Forward with reverse shocks, Astrophys. Space Sci., 120, 211-221.

Smith, Z., M. Dryer, C. D. Fry, W. Sun, and C. S. Deehr (2002), The role of interactions of solar-initiated interplanetary disturbances within $1 \mathrm{AU}$ in real time predictions of shocks, in Proceedings of SOLSPA-2, ESA SP-409, pp. 377-380, Eur. Space Agency, Paris.

Smith, Z., T. Detman, M. Dryer, C. D. Fry, C.-C. Wu, W. Sun, and C. S. Deehr (2004), Verification of space weather models that predict arrivals of interplanetary shocks at Earth based on solar data, IEEE Trans. Plasma Sci., 32, 1498-1505.

Veselovsky, I. S., et al. (2004), Solar and heliospheric phenomena in October-November 2003: Causes and effects, Cosmic Res., 42(5), $435-488$.

Whang, Y. C., and L. F. Burlaga (1986), The coalescence of two merged interaction regions between 6.2 and 9.5 AU: September 1979 event, J. Geophys. Res., 91, 13,341-13,348.

Winslow, A. M. (1966), Numerical Solution of the quasilinear Poisson equation in a nonuniform triangle mesh, J. Comput. Phys., 1, 149-172.

Wu, C.-C., M. Dryer, and S. T. Wu (1996a), Three-dimensional MHD simulation of interplanetary magnetic field changes at $1 \mathrm{AU}$ as a consequence of simulated solar flares, Ann. Geophys., 14, 383-399.

Wu, C.-C., S. T. Wu, and M. Dryer (1996b), Generation and evolution of interplanetary slow shock, Ann. Geophys., 14, 375-382.

Wu, C.-C., S. T. Wu, and M. Dryer (2004), Evolution of fast and slow shock interactions in the inner heliosphere, Sol. Phys., 223, 259-282.

Wu, C.-C., M. Dryer, and S. T. Wu (2005a), Slow shock interactions in the heliosphere using an adaptive grid MHD model, Ann. Geophys., 23, $1013-1023$.

Wu, C.-C., C. D. Fry, D. Berdichevsky, M. Dryer, Z. Smith, and T. Detman (2005b), Predicting the arrival time of shock passages at Earth, Sol. Phys., $227,371-386$.

Wu, S. T., T. X. Zhang, E. Tandberg-Hanssen, Y. Liu, X. Feng, and A. Tan (2004), Numerical MHD experiments for testing the physical mechanisms of coronal mass ejections, Sol. Phys., 225, 157-175

Zurbuchen, T. H., G. Gloeckler, F. Ipavich, J. Raines, C. W. Smith, and L. A. Fisk (2004), On the fast coronal mass ejections is October/ November 2003: ACE-SWICS results, Geophys. Res. Lett., 31, L11805, doi:10.1029/2004GL019461.

D. Berdichevsky, L-3 Communications EER Systems, Inc., 1801 McCormick Drive, Suite 170, Largo, MD 20774, USA. (xrdbb@lepvax. gsfc.nasa.gov)

M. Dryer, T. Detman, and Z. Smith, NOAA Space Environment Laboratory Center, R/E/SE, 325 Broadway, Boulder, CO 80305, USA. (murray.dryer@noaa.gov; thomas.detman@noaa.gov; zdenka.smith@ noaa.gov)

C. D. Fry, Exploration Physics International, Inc., Huntsville, AL 35806, USA. (gfry@expi.com)

N. Gopalswamy, NASA Goddard Space Flight Center, Greenbelt, MD 20771, USA. (gopals@fugee.nasa.gov)

R. Skoug, Los Alamos National Laboratory, MS D466, Los Alamos, NM 87545, USA. (rskoug@lanl.gov)

C. Smith, Institute for Earth, Oceans, and Space, University of New Hampshire, Durham, NH 03824, USA. (chuck@briaxa.sr.unh.edu)

C.-C. Wu and S. T. Wu, Center for Space Plasma and Aeronomic Research, University of Alabama, Huntsville, AL 35899, USA. (wuc@ cspar.uah.edu; wus@cspar.uah.edu)

T. Zurbuchen, Department of Atmospheric, Oceanic, and Space Sciences, University of Michigan, Ann Arbor, MI 48109, USA. (thomasz@umich. edu) 